\title{
Net gains
}

\author{
Physics - and physicists - have had much to contribute to economic and finance. Now the science of \\ complex networks sets a way forward to understanding and managing the complex financial networks \\ of the world's markets.
}

When H. Eugene Stanley coined the term 'econophysics' at a Kolkata conference in 1995, the field was still in its infancy despite having already spawned a slew of papers by statistical physicists, eager to lend their expertise to an intriguing new set of problems. This trend was born in the wake of a sudden availability of large amounts of financial data in the 1980s. But it may have had just as much to do with a frustration at the inadequacy of traditional theoretical approaches to economics, which seemed to favour model simplicity over accuracy, or agreement with empirical data.

Perhaps even more attractive for physicists, were the inherent mathematical similarities between market movements and the physical systems with which they were already familiar. The fundamental theorem of arbitrage-free pricing, for example, had given rise to the Black-Scholes equation for option pricing - a formula easily recognized as a type of diffusion-advection equation.

Early econophysics dealt primarily with these analogies - providing insight into the stochasticity and nonlinearity that characterize market dynamics. Physicists were able to shed some light on the origin of fat tails that were showing up in the distributions of financial data, deviating significantly from the Gaussian approximations of the BlackScholes formalism. These anomalous statistics were found to be a natural property of the universal scaling associated with market players exploiting microtrends in the data.

The proliferation of econophysicists lining the halls of university departments coincided with a flood of physicists leaving academia to enter the financial sector. Young mathematicians and physicists, equipped with little more than $\mathrm{PhD}$ and a quick wit, were recruited into an industry that was fast learning the value of minds well-versed in the language of partial differential equations.

So when the world at large fell into financial crisis in 2008, physicists were quickly identified by many as one of the key reasons behind the collapse. In an October 2008 interview on US

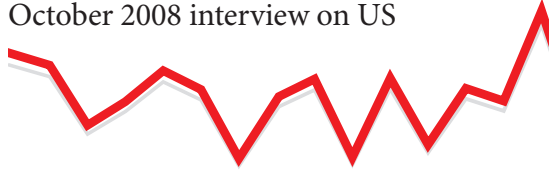

television's 60 minutes, Jim Grant founder of Grant's Interest Rate Observer, a twice-monthly journal of financial markets - blamed the mess on "mortgage science projects devised by these Nobeltracked physicists who came to work on Wall Street for the very purpose of creating complex instruments"1.

And Grant's certainly wasn't a lone voice. Some even went as far as to $\operatorname{argue}^{2}$ that the prevalence of Asperger's syndrome in the physics community was responsible for the crisis - that physicists working in the financial sector weren't capable of feeling empathy for the lives that stood to be ruined from the inevitable failure of their complex models.

In reality, the collapse was attributable to a combination of factors. Investment houses that had purchased countless mortgages of questionable reliability turned to their quantitative minds in-house to divide up and repackage the debts as exotic new products. This process was, however, designed to minimize risk - providing safe investments with above-average returns. The argument can be made that the subsequent judgment calls of executives and managers (armed with information they did not understand) were more dangerous than the instruments themselves.

Despite the misgivings of the popular media, and the relative downturn in the employment prospects of the financial sector, the trend for physicists to enter the industry has not abated - nor, it must be said, has momentum for physicists in academia to turn their attention to problems associated with market dynamics. Indeed, the quantitative finance $\operatorname{archive}^{3}$ was launched in December 2008, largely to service a wealth of submissions that was being distributed amongst the existing fields.

In recent years, however, the focus of these efforts has shifted towards the realm of network science. This new endeavour addresses a need to understand the structure
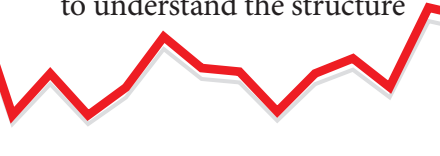

and dynamics underlying financial markets, to explain - and anticipate - the effects that interactions between many agents are capable of inducing. An optimistic view is that, equipped with such knowledge of systemic behaviour, we might even be able to influence market dynamics using the tools of complex networks science.

One practical way in which this might be achieved involves recognizing the financial institutions that are too important to system stability to risk allowing their failure. The popular notion of a bank being too big to fail is currently being revised by scientists with an intimate knowledge of how networks function. In this Focus issue of Nature Physics, we have drawn together a sequence of Commentaries from physicists working in close quarters with prominent economists to elaborate on this, and other related directions, in the statistical physics of complex financial networks.

A report commissioned by the Centre for the Study of Financial Innovation in the late 1990s described the financial sector as "depressingly unenthusiastic" about the insight emerging from econophysics research conducted in university departments. The general feeling seemed to be that econophysicists' desire to understand the underlying dynamics of the market was fundamentally at odds with traders'

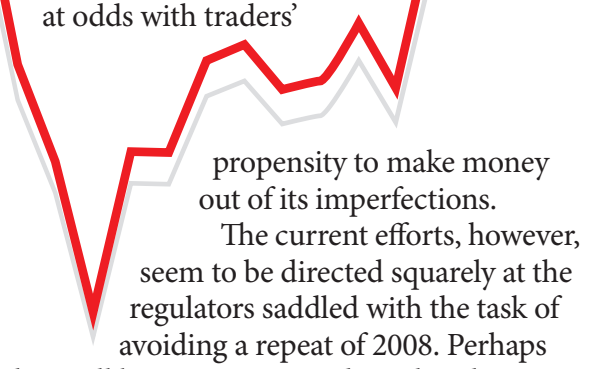

they will be more interested in what the physicists have to say than were the market players of the late twentieth century.

\footnotetext{
References

1. http://www.youtube.com/watch?v=2mphjxFYigg

2. Luyendijk, J. The Joris Luyendijk Banking Blog. The Guardian (8 May, 2012); available at http://go.nature.com/9PZKtC

3. http://arxiv.org/archive/q-fin

4. Lascelles, D. Quant and Mammon, Meeting the City's Requirements for Post-graduate Research and Skills in Financial Engineering (Centre for the Study of Financial Innovation, 1999).
} 